\title{
Dumping STAT3 in the trash
}

Induced protein degradation can potentially be used to therapeutically

SD-36mediated STAT3 degradation is highly selective over $\sim 5,500$ proteins ... these data highlight the potential for targeted degradation approaches to have greater selectivity than those based on direct target inhibition g modulate previously 'undruggable' targets, such as transcription factors. In Cancer Cell, Bai and colleagues now report a proteolysis targeting chimaera (PROTAC) for signal transducer and activator of transcription 3 (STAT3), an attractive but challenging cancer target. This PROTAC elicits potent and selective STAT3 degradation, resulting in complete tumour regression in mouse models of blood cancers.

Previous efforts to target STAT3 have focused on inhibiting its canonical activation process, which involves dimerization mediated by the reciprocal interactions between a key phosphorylated tyrosine residue (Y705) and the $\mathrm{SH} 2$ domain. However, as well as being highly challenging to develop a selective inhibitor of this interaction, such compounds are unlikely to fully block STAT3 transcriptional activity, which can also be mediated by the

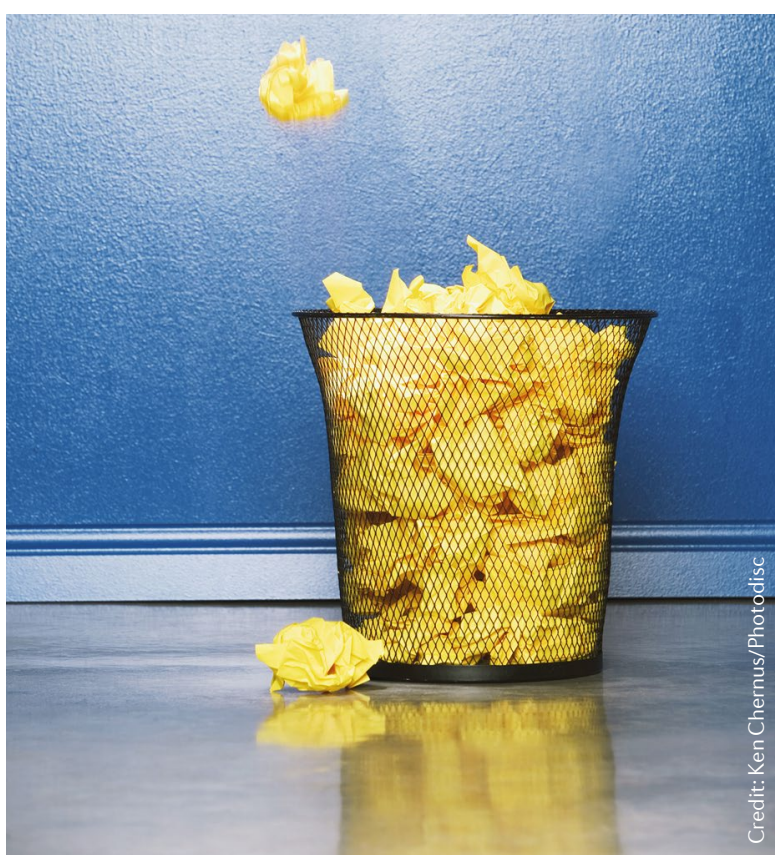

monomeric form of the protein for some genes.

Bai and colleagues first developed a cell-permeable PROTAC called SD-36 $\left(K_{\mathrm{i}}=11 \mathrm{nM}\right)$ by optimizing their previously reported STAT3 $\mathrm{SH} 2$ domain inhibitor. After $7 \mathrm{~h}$, SD-36 $(0.25 \mu \mathrm{M})$ promoted $\geq 50 \%$ STAT3 degradation in leukaemia and lymphoma cell lines through a ubiquitin-proteasome-dependent mechanism. Mutating residues key for SD-36 binding within the STAT3 SH2 domain identified from crystallographic data rendered these STAT3 variants resistant to degradation, confirming the mode of action. Importantly for its therapeutic relevance though, SD-36 still promoted the degradation of STAT3 variants with somatic mutations that are present in several haematological malignancies, as the mutated residues are not key for SD-36-STAT3 binding.

SD-36 showed modest binding selectivity for STAT3 over other STATs in vitro ( 20 -fold over STAT1 and STAT4), probably owing to the high structural homology of the $\mathrm{SH} 2$ domain among the STAT proteins. However, in proteomics studies using human peripheral blood mononuclear cells, the authors observed that SD-36-mediated STAT3 degradation is highly selective over $\sim 5,500$ proteins, including the remaining STATs. These data highlight the potential for targeted degradation approaches to have greater selectivity than those based on direct target inhibition.

Mechanistically, SD-36-mediated STAT3 degradation depleted cells of both monomeric and dimeric STAT3, and abrogated downstream DNA binding and transcriptional activity. Using RNA sequencing, the authors observed attenuated transcription of downstream STAT3 target genes such as BCL3, JAK3 and SOCS3, further supporting the specificity of SD-36 for STAT3.

When evaluated in various cell lines, SD-36 attenuated growth in only a subset of leukaemia and lymphoma cell lines. Most of the susceptible cells normally express high levels of pSTAT3, and SD-36 treatment reduced levels of Myc, a STAT3-dependent mediator of oncogenic signalling. Further analysis revealed that $\mathrm{SD}-36$ promoted $\mathrm{G}_{1}$ arrest, thereby reducing the number of $S$ phase cells, and upregulated apoptotic cell death.

In vivo, treatment with SD-36 intravenously resulted in complete and long-lasting tumour regression in both leukaemia and lymphoma xenograft models. Meanwhile, SD-36 $(100 \mathrm{mg} / \mathrm{kg})$ was well-tolerated in immunocompetent mice in which STAT3 was depleted in liver, spleen, heart and kidney. No major changes were observed in blood chemistry, suggesting that no harmful anti-SD-36 immune responses occurred.

Overall, the study indicates that targeted degradation of STAT3 may be a promising strategy to surpass the limited clinical activity of STAT3 inhibitors observed to date. Encouragingly, no problems have been reported for the first two PROTACs to enter the clinic so far, and the authors of the study highlighted here say that a STAT3-targeted PROTAC could be advanced into clinical development as early as 2020 .

Stacey-Lynn Paiva

ORIGINAL ARTICLE Bai, L. et al. A potent and selective small-molecule degrader of STAT3 achieves complete tumor regression in vivo. Cancer Cell 36, 498-511 (2019) RELATED ARTICLES Schapira, M. et al. Targeted protein degradation: expanding the toolbox. Nat. Rev. Drug Discov. https://doi.org/10.1038/ s41573-019-0047-y (2019) | Mullard, A. Arvinas's PROTACs pass first safety and PK analysis. Nat. Rev. Drug Discov. https://doi.org/10.1038/d41573-01900188-4 (2019) 